\title{
PION-MUON CONCENTRATING SYSTEM FOR DETECTORS OF HIGHLY ENRICHED URANIUM*
}

\author{
S. Kurennoy, F. Neri, D. Barlow, B. Blind, and A. Jason, LANL, Los Alamos, NM 87545, USA
}

\section{Abstract}

One of many possible applications of low-energy antiprotons collected in a Penning trap can be a portable muon source. Released antiprotons annihilate on impact with normal matter producing on average about three charged pions per antiproton, which in turn decay into muons. Existence of such negative-muon sources of sufficient intensity would be useful for detection and enrichment determination of highly enriched uranium based on muonic X-rays. We explore options of collecting and focusing pions and resulting muons to enhance the muon flux toward an object of interest. Simulations with MARS and MAFIA are used to choose the target material and parameters of the magnetic system of a few solenoids.

\section{INTRODUCTION}

A transportable antiproton bottle (Penning trap) can contain up to $\sim 10^{12}$ pbars. Released antiprotons annihilate on impact with matter producing pions with an isotropic spatial distribution. On average, about three charged pions per annihilation are produced, with average kinetic energy of about $250 \mathrm{MeV}$. Charged pions then decay into muons plus (anti)neutrinos with a mean lifetime $\tau_{0}=26 \mathrm{~ns}$ in their rest frame. The negative muons produced are of special interest. Due to their strong penetrating ability, one can detect highly enriched uranium (HEU) inside containers based on specific signatures of muonic X-rays [1]. Since the number of trapped antiprotons is limited, a system that can increase the muon flux toward a target by capturing and focusing negative pions and muons is desirable.

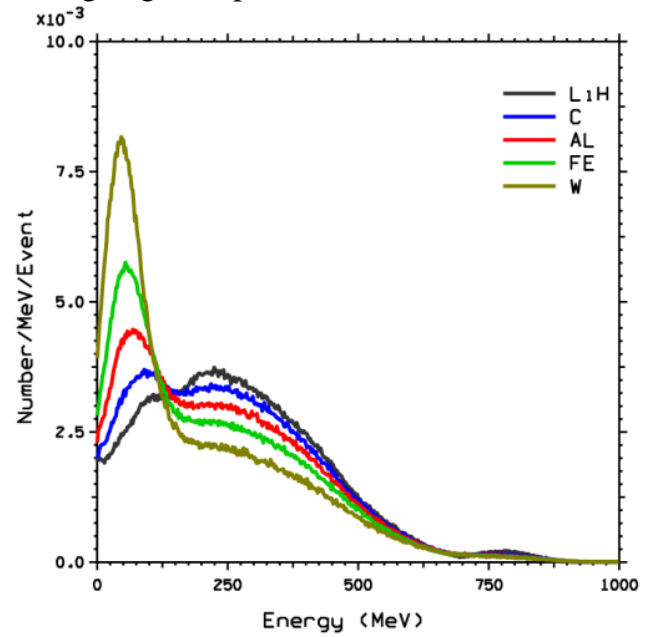

Figure 1: Pion spectra for different target materials.

\section{ANNIHILATION PION SPECTRA}

Kinetic-energy spectra of $\pi$ s produced in antiproton annihilations at rest on targets made of different materials

\footnotetext{
*Work supported by the US DOE under contract W7405-ENG-36.
}

and calculated [2] with the Monte Carlo code MARS [3] are plotted in Fig. 1. Experimental data on nuclei are sketchy, at best. The $\pi^{-}$-multiplicity is almost the same for different materials, about 1.5 , but the fraction of lowenergy pions is noticeably higher for heavy metals. The number of produced neutrons also increases significantly for heavy-material targets, becoming almost an order of magnitude higher for tungsten (W) than for carbon (C).

The choice of target is defined by the need to capture pions and produce muons in a certain energy range. Lowenergy pions are easier to capture, but they lack in penetrating abilities, see below. This requirement favors light materials like $\mathrm{LiH}$ or $\mathrm{C}$ because of a larger fraction of pions produced with higher energies.

\section{COLLECTION AND FOCUSING SYSTEM}

\section{System Requirements.}

A few considerations should be taken into account:

1. The number of particles at a given energy decreases $e$-fold over the path length $\beta \gamma l_{0}$, where $l_{0}=c \tau_{0}$. For charged pions $l_{0}=7.8 \mathrm{~m}$, for muons $658.7 \mathrm{~m}$. The distance from an annihilation target to muon targets should be neither too short (>10-20 m), nor too long (below a few hundred $\mathrm{m}$ ).

2. Ionization energy losses of muons in matter are minimal (and penetration maximal) for muon kinetic energies $T=100-400 \mathrm{MeV}$ (momenta $200-500 \mathrm{MeV} / \mathrm{c}$ ). At lower energies, the losses are high, so that a $10-\mathrm{MeV}$ muon, for example, will be stopped in a few meters of air.

3. An angle between the directions of an initial pion and the muon produced by its decay, $\pi^{-} \rightarrow \mu^{-}+\tilde{v}_{\mu}$, is rather small in the lab frame for the pion energies of interest: e.g., below $7.85^{\circ}$ for a typical pion momentum of 300 $\mathrm{MeV} / \mathrm{c}(\beta=0.9, T=190 \mathrm{MeV})$, see [4] for detail.

4. Capturing charged pions with transverse momentum $p_{\mathrm{t}}$ in a solenoidal field $B_{\mathrm{z}}$ requires the aperture radius of a magnetic system $R_{\mathrm{a}}>2 R$, where $R$ is given by $B_{z} R=p_{t} / q$ ( $q$ is the charge). For $p_{\mathrm{t}}=300 \mathrm{MeV} / \mathrm{c}$ pions, $B_{z} R \simeq 1 \mathrm{~T} \cdot \mathrm{m}$.

5. Any mobility requirements limit the system size and weight. For example, to be transported by a semi-trailer, the system length and width are limited to $30 \mathrm{ft}(10 \mathrm{~m}) \times 8$ $\mathrm{ft}(2.4 \mathrm{~m})$, and the weight should be below 20 tons.

Now we define requirements to the collection system. With a goal to enhance the muon flux toward the detector by at least one order of magnitude, the system should:

- Deliver antiprotons to the annihilation target, while shielding the antiproton bottle from the magnetic field.

- Capture produced pions with transverse momenta as high as possible (up to $500 \mathrm{MeV} / \mathrm{c}$ ).

- Reduce the divergence of the exiting beam of muons and remaining pions (ideally, produce a parallel beam). This is difficult, because the beam energy spread is large. 


\section{Magnetic Field Configurations. Solenoids.}

For collecting and focusing the produced pions, two magnetic field configurations are suitable: (i) a decreasing solenoidal field, and (ii) an azimuthal field around the target (horn). Approach (i) is proposed for the Muon Collider project [5]: solenoids with gradually increasing apertures create a strong magnetic field that decreases downstream from the target. Approach (ii) has long been used at CERN in neutrino studies, and is now planned for the CERN neutrino factory [6]. The azimuthal magnetic field is created inside a hollow thin-wall metallic body by axisymmetric wall currents looped in $r-z$ planes. In both examples, a proton beam with the energy of tens of $\mathrm{GeV}$ produces pions mostly in the forward direction. Our case, where antiprotons annihilate practically at rest, is very different, since pion momenta are directed isotropically.

First, we consider a model axisymmetric magnetic field $B_{z}(z, r)=B_{0} f(z), B_{r}(z, r)=-B_{0} f^{\prime}(z) r / 2$, where $f(z)$ is a piecewise function: linear $1-z / L$ for $0<z<L_{1}$, it vanishes quadratically for $L_{1}<z<L_{2}$. With $L=\left(L_{1}+L_{2}\right) / 2$, we have a continuous field that decreases linearly and then decays, cf. [4]. Choosing solenoid length $L=5 \mathrm{~m}$, aperture $R=1 \mathrm{~m}$, the field decay length $L_{2}-L_{1}=2 R=2 \mathrm{~m}$, we take $L_{1}=4 \mathrm{~m}$, $L_{2}=6 \mathrm{~m}$. Equations of motion in this field are integrated with Mathematica. As an example, Fig. 2 shows the radial positions of pions with $T=190 \mathrm{MeV}$ versus $z$ for $B_{0}=4 \mathrm{~T}$ and for initial pion axial angles $\theta_{\mathrm{z}}=10^{\circ}, 20^{\circ}, \ldots, 80^{\circ}$.

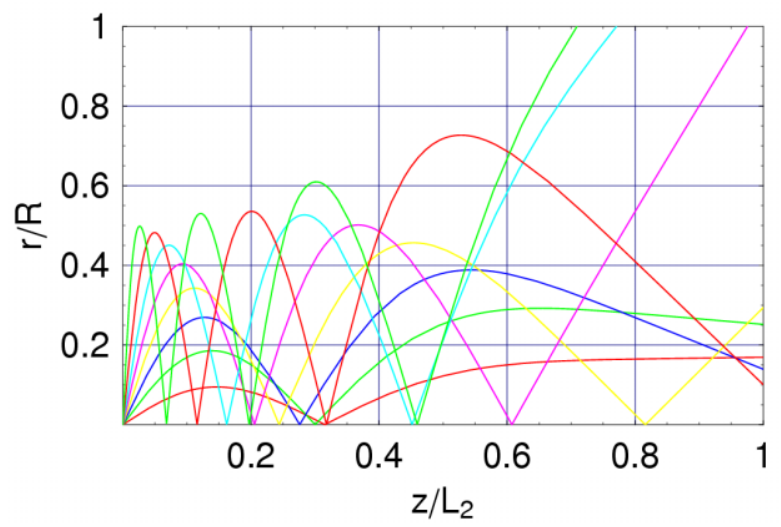

Figure 2: Pion radial position versus longitudinal.

The pions with $\theta_{\mathrm{z}}=60^{\circ}$ and $80^{\circ}$ hit the wall, while those with $\theta_{\mathrm{z}}=70^{\circ}$ and below $50^{\circ}$ exit the system. Such a nonmonotonic dependence of the exit angle on the initial is confirmed by Fig. 3. As one can see in Fig. 3, results of particle tracking with MARS [4] in the model field (stars for pions) agree well with the analytical results from Mathematica (curves). Dashed curve parts correspond to particles intercepted by the aperture. MARS data are from tracking in air, but the angles in vacuum are almost identical. MARS also tracks muons (diamonds) produced by pion decays: their angles deviate randomly, but by a small amount, from those of parent pions. Due to that reason some muons pass through the system even when their parent pions were in the "intercept-angle" zones (dashed parts near $\theta_{\mathrm{in}}=60^{\circ}$ and $80^{\circ}$ ).

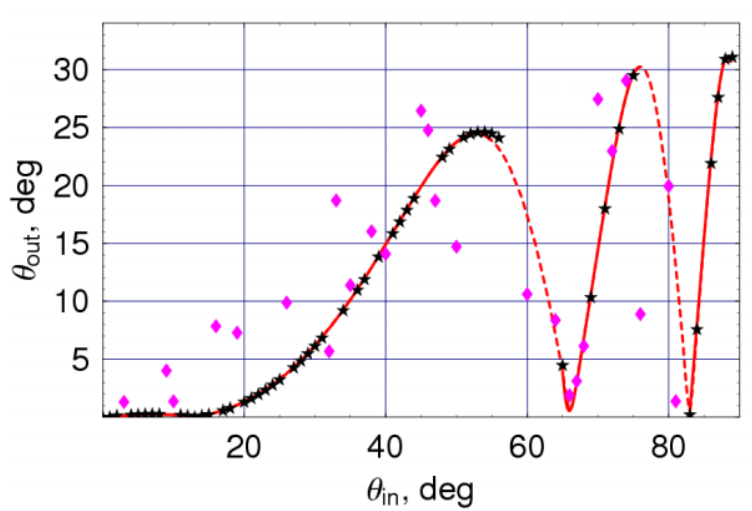

Figure 3: Pion exit angle versus its initial angle for $B_{0}=4 \mathrm{~T}$, $T=190 \mathrm{MeV}$. MARS results (pions - stars, muons diamonds) compared with analytical predictions (curves).

We proceed with two realistic magnetic systems. Both are 5-m long and have five solenoidal magnets of various lengths: (I) with large apertures and lower field (Fig. 4), and (II) small apertures but stronger field. For system I, the smallest magnet radius is $0.5 \mathrm{~m}$, the largest $0.9 \mathrm{~m}$, and the maximal on-axis field is $B_{0}=4 \mathrm{~T}$. Steel C1006 is used for shielding (red in Fig. 4), and the field is calculated by MAFIA taking into account the steel nonlinear magnetic properties. Figure 4 plots the arrows of the flux density $B$ in $(z, r)$-plane ( $z$-axis is horizontal). The magnet currents are chosen to provide a decreasing field profile similar to that of the model field above. The steel is configured on the upstream end to shield the antiproton bottle and to clamp the field forming its peak near the annihilation target - a tiny green rectangle near $(0,0)$.

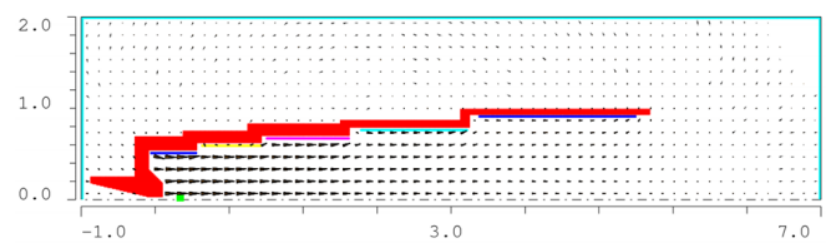

Figure 4: Magnetic field arrows. Dimensions are in m. Magnet coils are shown by color lines inside the shield.

First, we use MAFIA particle-in-cell module TS2 to study propagation of pions. With an approximate energy spectrum - a flattop distribution of pion velocities from $0.55 c$ to $0.965 c(T=25-400 \mathrm{MeV})$ - the capture fraction, defined as the ratio of the number of pions exiting the magnet to the total initial number of pions, is 0.40 .

Realistic simulations must account for pion decays and an accurate energy spectrum. We use MARS for such simulations, mapping the MAFIA-calculated fields into it. Annihilations of $N_{\mathrm{pb}}=10^{5}$ antiprotons in a thin $\mathrm{LiH}$ target give $N=154510 \mu$-producing events (mostly $\pi^{-}$, and a few $\left.K^{\prime}\right)$ [2]. The code monitors pions and muons at various locations after the magnets. Some results are summarized in Tab. 1 , where $N_{\pi \mu}=N_{\pi}+N_{\mu}$, and $N_{\mu} / N_{\mathrm{pb}}$ is the number of muons per antiproton. The capture fraction is 0.265 , i.e. $26.5 \%$ of pions (and decay muons) are captured. This is $0.41\left(\pi^{-}+\mu^{-}\right)$per incident antiproton. The same MARS simulations in vacuum instead of air give a higher capture 
rate, $34.3 \%$. The difference is due to low-energy muons and pions stopped in air by ionization losses. It is lower than our estimate from TS2 simulations, mainly because of differences in the pion spectra used by two codes. The enhancement factor $X$ in Tab. 1 is defined as the ratio of pion-muon fluxes with the magnet system and without it, through the same target. The expected flux without magnets is $\sim N(R / L)^{2} / 4$ for an object of radius $R$ at distance $L$ when $L \gg R$. System I enhances the flux for small targets at large distances by a factor of about 40 .

Table 1: Particle capture \& fluxes w/o and with horn (h)

\begin{tabular}{|l|c|c|c|c|c|}
\hline Monitor & $N_{\pi \mu} / N$ & $N_{\pi \mu} / N, \mathbf{h}$ & $X$ & $X, \mathbf{h}$ & $N_{\mu} / N_{\mathbf{p b}}, \mathbf{h}$ \\
\hline Magn. exit & 0.265 & 0.265 & 35.5 & 35.5 & 0.143 \\
\hline 20m, $r<2 \mathrm{~m}$ & 0.066 & 0.083 & 26.4 & 33.3 & 0.088 \\
\hline 20m, $r<1 \mathrm{~m}$ & 0.027 & 0.035 & 42.7 & 55.5 & 0.034 \\
\hline 30m, $r<2 \mathrm{~m}$ & 0.033 & 0.040 & 29.5 & 36.2 & 0.049 \\
\hline 30m, $r<1 \mathrm{~m}$ & 0.011 & 0.014 & 39.4 & 49.2 & 0.015 \\
\hline
\end{tabular}

Tracks of pions and muons from MARS simulations with a $\mathrm{LiH}$ target in air are shown in Fig. 5. The region is a cut in $(y, z)$-plane, $-1 \mathrm{~m}<z<10 \mathrm{~m}$, scale $y: z=1: 1$. Mesh lines show the MARS region subdivision, and have steps of $1 \mathrm{~m}$ in $z$ in the magnet region, and $0.5 \mathrm{~m}$ in $r$. A simplified shielding layout with the correct aperture profile and wall thickness was used.

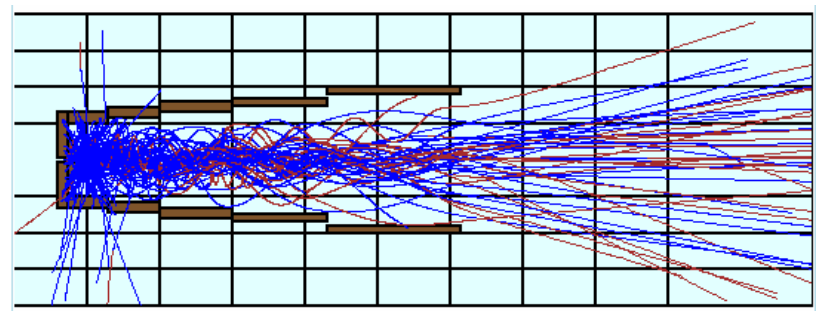

Figure 5: MARS: tracks for 200 initial pions in air. Pions are in blue, muons in red, and the shielding is brown.

A similar magnet system II, which has smaller apertures ( $R=0.25 \mathrm{~m}$ to $0.5 \mathrm{~m}$ ) and 8 -T peak field, gives a lower capture fraction, $17.8 \%$ in air. Still, it produces 0.28 $\left(\pi^{-}+\mu^{-}\right)$per antiproton, and $X$ s are around 30.

For both systems, the exiting beam has a wide angular distribution and energy spread. For system I, the average angle $\theta_{\mathrm{z}}=9.6^{\circ}$ and $11.2^{\circ}$, while average kinetic energy $T=241$ and $151 \mathrm{MeV}$, for pions and muons, respectively. We try to focus the beam further by placing a magnetic reflector (horn) after the solenoids.

\section{Magnetic Horn-Reflector after Solenoids}

We choose the horn azimuthal magnetic field to kick particles with the average values of the angle and energy to zero-slope trajectories. Our horn cross section is an inverted trapezoid with its small base $s_{0}$ equal to the inner radius $r_{0}=0.35 \mathrm{~m}$, the outer radius $r_{1}=s_{1}=1 \mathrm{~m}$, and the angle $51.3^{\circ}$ between the sidewalls, cf. Fig. 6 . The required field is $0.5 \mathrm{~T}$ at the inner radius; it decreases as $1 / r$ inside the horn shell. Such a horn provides the same angular kick to all particles with a given momentum independent of the radial position of their passage through the horn (at small angles) [4]. Its effect is illustrated in Fig. 6 and in Tab. 1 (columns marked ' $h$ '). For system I, the horn increases the enhancement factors by $20-30 \%$, to about 50 at large distances. A similar horn in system II increases the fluxes by $30-40 \%$, bringing $X$ s to about 40, see [4] for detail.

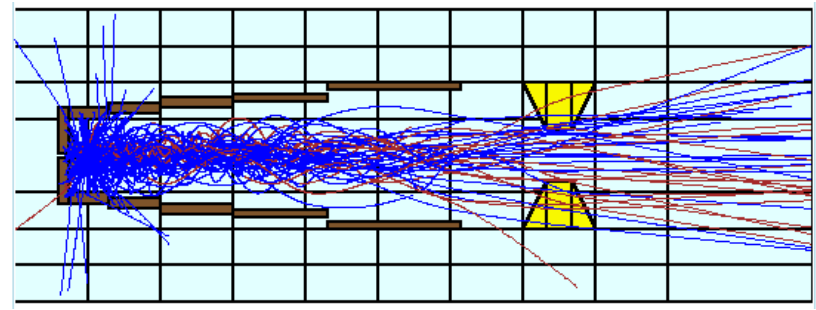

Figure 6: Same as Fig. 5, with magnetic horn (yellow).

\section{CONCLUSIONS}

We have explored some options of collecting and focusing negatively charged pions created by antiproton annihilations, as well as muons produced by pion decays, to enhance the muon flux toward the detector. The considered system of pulsed solenoidal magnets followed by a magnetic horn-reflector enhances the muon flux by a factor of 50 compared to a spatially isotropic distribution. Solenoids also increase the pion path length and, hence, their decay probability. An optimization can likely bring the flux enhancement to at least two orders of magnitude.

About $2.5 \cdot 10^{7}$ muons are required on a $10-\mathrm{kg}$ uranium target with a $5-\mathrm{cm}$ lead shielding (one of the worst cases) to get $\sim 100$ muonic X-ray counts - sufficient to determine the uranium enrichment with the accuracy of $10 \%$ [1]. Using $N_{\mu} / N_{\text {pb }}$ from Tab. 1 at $z=30 \mathrm{~m}, r<1 \mathrm{~m}$, and assuming $0.1-\mathrm{m}$ radius for such a target, for $10^{12}$ stored antiprotons in the bottle one expects about $1.5 \cdot 10^{8}$ muons delivered to this sample by the system.

The authors would like to thank N.V. Mokhov (FNAL) for his help with the MARS code and for adapting the code to our case of very low energy antiprotons.

\section{REFERENCES}

[1] M.W. Johnson, Private communication, Los Alamos, 2002.

[2] F. Neri, LANSCE-1: 04-16, Los Alamos, 2004.

[3] N.V. Mokhov et al, "Recent Enhancements to the MARS15 Code," Fermilab-Conf-04/053, 2004.

[4] S. Kurennoy, Tech. note LANSCE-1: 05-016 (TN), Los Alamos, 2005.

[5] C.M. Ankenbrandt et al. Phys. Rev. ST-AB, 2 (1999) 081001.

[6] S. Gilardoni et al. J. Phys. G: Nucl. Part. Phys. 29 (2003) 1801. 